\title{
C2 Fractures in the Elderly: Single-Center Evaluation of Risk Factors for Mortality
}

\author{
Hoi-Ying H. Chan ${ }^{1}$, Frank A. Segreto ${ }^{2}$, Samantha R. Horn ${ }^{2}$, Cole Bortz ${ }^{2}$, \\ Godwin G. Choy ${ }^{1}$, Peter G. Passias ${ }^{2}$, Hamish H. Deverall ${ }^{1}$, Joseph F. Baker ${ }^{1,3}$ \\ ${ }^{1}$ Department of Orthopaedic Surgery, Waikato Hospital, Hamilton, New Zealand \\ ${ }^{2}$ Department of Orthopaedic and Neurological Surgery, NYU Langone Medical Centre-Orthopaedic Hospital, New York, NY, USA \\ ${ }^{3}$ Department of Surgery, University of Auckland, Auckland, New Zealand
}

Study Design: Retrospective cohort study.

Purpose: The aim of this study was to identify features associated with increased mortality risk in traumatic C2 fractures in the elderly, including measures of comorbidity and frailty.

Overview of Literature: $\mathrm{C} 2$ fractures in the elderly are of increasing relevance in the setting of an aging global population and have a high mortality rate. Previous analyzes of risk factors for mortality have not included the measures of comorbidity and/or frailty, and no local data have been reported to date.

Methods: This study comprises a retrospective review of 70 patients of age $>65$ years at Waikato Hospital, New Zealand with traumatic C2 fractures identified on computed tomography between 2010 and 2016. Demographic details, medical history, laboratory results on admission, mechanism of injury, and neurological status on presentation were recorded. Medical comorbidities were also detailed allowing calculation of the Charlson Comorbidity Index (CCI) and the modified Frailty Index (mFI).

Results: The most common mechanism of injury was a fall from standing height $(n=52,74.3 \%)$. Mortality rates were $14.3 \%(n=10)$ at day 30 , and $35.7 \%(n=25)$ at 1 year. Bivariate analysis showed that both $\mathrm{CCl}$ and $\mathrm{mFl}$ correlated with 1 -year mortality rates. Reduced albumin and hemoglobin levels were also associated with 30-day and 1-year mortality rates. Forward stepwise logistic regression models determined $\mathrm{CCl}$ and low hemoglobin as predictors of mortality within 30 days, whereas $\mathrm{CCl}$, low albumin, increased age, and female gender predicted mortality at 1 year.

Conclusions: The $\mathrm{CCl}$ was a useful tool for predicting mortality at 1 year in the patient cohort. Other variables, including common laboratory markers, can also be used for risk stratification, to initiate timely multidisciplinary management, and prognostic counseling for patients and family members.

Keywords: Fracture; Axis; Mortality; Charlson Comorbidity Index; Modified Frailty Index

\section{Introduction}

$\mathrm{C} 2$ is the most commonly injured vertebra in the cervical spine in those aged $>65$ years [1]. While the majority of
C2 fractures in elderly patients are caused by a fall from standing height, the implication of this injury for the individual is far from simple. Associated mortality and morbidity rates are significant, with 1-year mortality rates

\footnotetext{
Received Nov 26, 2018; Revised Jan 30, 2019; Accepted Jan 31, 2019

Corresponding author: Hoi-Ying H. Chan

Department of Orthopaedic Surgery, Whangarei Hospital, Maunu Road, Private Bag 9742, Whangarei 0148, New Zealand

Tel: +64-21-029-38554, Fax: +64-9-470-0088, E-mail: heidi.chan@live.com
} 
as high as $37.5 \%$ having been reported [2], comparative to those of neck of femur fractures, indicating a potential end-of-life event for vulnerable elderly patients. With an aging population globally, it is expected that the incidence of these fractures will continue to rise.

Previous studies focusing on odontoid fractures in the elderly have attempted to ascertain risk factors associated with mortality and poor outcome. In a recent retrospective review of 83 elderly patients, Bajada et al. [3] established predictive risk factors, including low serum hemoglobin, neurological deficit on presentation, admission from an institution, and type 3 odontoid fractures for 30day and 1-year mortality. Tashjian et al. [4] also found that immobilization with a halo-vest significantly increased mortality rate. The identification of such associations is clinically important for risk stratification, treatment decision making, and providing meaningful outcome counseling for patients and their family.

Similar observations have been the focus of investigations on neck of femur fractures in the elderly, whereby comorbidities have been shown to correlate with increased mortality rate and poorer outcomes. In particular, the Charlson Comorbidity Index (CCI) has remained a popular predictive scoring tool to represent the disease burden on a patient in both clinical and research settings $[5,6]$. However, whether this is a significant predictor of mortality rates in $\mathrm{C} 2$ fractures of the elderly remains to be elucidated. In recent years, frailty has also become an increasingly recognized concept to define elderly vulnerability and dependence as a separate entity to comorbidity and disability $[7,8]$. The modified Frailty Index (mFI), developed from the Canadian Study of Health and Aging Frailty Index, has been shown to be a reliable tool for preoperative risk stratification in specialties including neurosurgery, cardiovascular surgery [9-11], and spinal surgery $[12,13]$.

The present study aimed to establish the 30-day and 1 -year mortality rates for elderly patients with traumatic C2 fractures treated at Waikato Hospital, New Zealand and to identify risk factors for mortality at these time points. CCI and mFI were included to determine their potential association with mortality rates.

\section{Materials and Methods}

A retrospective review was performed of all patients aged $\geq 65$ years with acute $\mathrm{C} 2$ fractures identified on computed tomography (CT) scans between 2010 and 2016 from the radiology trauma database. This retrospective outcomes analysis was approved by the Waikato Hospital Clinical Audit Support Unit (ref: 3783) and the requirement for informed consent waived. The analysis was performed in compliance with the Health Information Privacy Code 1994. Pathological fractures secondary to tumor, infection, or metastatic disease were excluded.

\section{Demographics}

Hospital electronic and paper records were reviewed to collect demographic information, including age, gender, and ethnicity, in addition to admission details. Previous medical history and mental (presence of dementia or cognitive impairment) and functional status prior to injury were recorded to enable calculation of the CCI and mFI.

\section{Charlson Comorbidity Index and modified Frailty Index}

The CCI places different weights on age brackets, selected disease categories and the severities of diseases to predict mortality rate, originally presented as an estimated 10year survival rate. For example, a CCI of $\geq 5$ estimates a 10 -year survival rate of $21 \%$, whereas a score of 2 predicts a 10 -year survival rate of $90 \%$ [14]. The $\mathrm{mFI}$ is an 11-item score, which divides the number of conditions or functional/mental deficits present by 11 , resulting in an index value between 0 and 1 for the patient to be classified as 'robust,' 'pre-frail', or 'frail.' The closer the value is to 1 , the frailer the individual [15]. The exact variables used to calculate both these scores are presented in Table 1 .

\section{Biochemical markers}

Serologic results on admission, including lymphocyte count, creatinine, albumin, and hemoglobin levels, were collated. Creatinine, albumin, and lymphocyte counts have all been linked to the presence of malnutrition and are thus considered to be a potential predictor of poor outcome [16-18].

\section{Other variables}

Details on the mechanism of injury and presence of neurological symptoms on admission were also recorded. 
Table 1. Individual variables and weights of clinical conditions or situations used to calculate the Charlson Comorbidity Index and modified Frailty Index, respectively

Condition (or history of)
Charlson Comorbidity Index
Age $50-59$ yr; coronary artery disease; congestive heart failure; chronic pulmonary disease; peptic ulcer disease; peripheral vascu-
lar disease; mild liver disease; cerebrovascular disease; connective tissue disease; diabetes mellitus; dementia
Age 60-69 yr; hemiplegia; moderate to severe renal disease; diabetes with end-organ damage; localised solid tumour; leukaemia;
lymphoma
Age $70-79$ yr; moderate to severe liver disease
Age $\geq 80$ yr
Metastatic solid tumour; acquired immune deficiency syndrome (not only human immunodeficiency virus positive)
Score
Modified Frailty Index
Diabetes mellitus; congestive heart failure; hypertension requiring medication; transient ischaemic attack; cerebrovascular acci-
dents/stroke with neurological deficit; impaired functional status (require home help, or in residential care); myocardial infarction
within 6 months; peripheral vascular disease or rest pain; acute or chronic lung diseases; impaired sensorium; cardiac history of
angina, prior cardiac surgery, or percutaneous cardiac intervention
Score

The types of $\mathrm{C} 2$ fractures were classified into Anderson and D'Alonzo types I, II, \& III for odontoid fractures, and others for Hangman's or atypical variant fractures. Severity was also considered, with fracture displacement determined by angulation and translation on CT where applicable, and associated fractures or injuries (e.g., femur and pelvic fractures) were recorded. Finally, treatment modality was recorded for descriptive analysis. All CT scans were reviewed by the lead and senior authors to determine the fracture type and displacement prior to the collection of other data. The decision regarding treatment strategy, whether nonsurgical or surgical, was made by the responsible spine surgeon at the time of admission.

All available data for up to 1 year post-injury were reviewed. The outcome measures comprised 90-day readmission rate, length of stay (LOS), and mortality rates at both 30 days and 1 year.

\section{Statistical analysis}

Pearson bivariate analysis was used to gage the associations between CCI, mFI, and serologic tests with 90-day readmission rates, LOS, and 30-day and 1-year mortality rates. Forward stepwise logistic regression modeling was then used to determine significant predictors of mortality within 30 days and 1 year of admission among the covariates of age, gender, $\mathrm{mFI}, \mathrm{CCI}$, hemoglobin, lymphocyte count, creatinine, and albumin levels. All $p<0.05$ were considered to indicate a statistically significant difference.

\section{Results}

\section{Descriptive analysis}

Following the exclusion of one pathological fracture, a total of 70 patients with traumatic $\mathrm{C} 2$ fractures were included in the data collection. The mean age at presentation was $80.6 \pm 8.5$ years, with the oldest patient aged 97 years; $60 \%$ of patients were female $(n=42)$, and the majority were of European descent $(88 \%, n=62)$.

The most common mechanism of injury was falling from standing height $(74.3 \%, \mathrm{n}=52)$, and $8 \%(\mathrm{n}=11)$ presented with abnormal neurological symptoms or signs. The majority of patients were treated nonsurgically, with either a hard or soft collar, with $11.4 \%$ undergoing some form of surgical stabilization or fixation $(n=8)$.

The mean CCI among all patients was 5 , and the mean mFI was 0.21 (pre-frail). The mean inpatient LOS was $10.4 \pm 12.5$ days, and 20 patients (28.5\%) were re-admitted to hospital within 3 months. The mortality rates were $14.3 \%(n=10)$ at 30 days and $35.7 \%(n=25)$ at 1 year (Table 2).

\section{Bivariate analysis}

Pearson's correlation results are shown in Table 3. In- 
creased CCI correlated linearly with both 30 -day $(R=0.367$, $p=0.002)$ and 1 -year $(R=0.690, p<0.001)$ mortality rates. Increased $\mathrm{mFI}$ also correlated with both 30 -day $(R=0.313$, $p=0.008)$ and 1 -year $(R=0.583, p=0.005)$ mortality rates, and with readmission rate within 90 days $(R=0.331$, $p=0.005)$. There was no association between a classification of 'robust' $(\mathrm{mFI}=0)$ or 'pre-frail' $(\mathrm{mFI}=0.09-0.18)$ with increased mortality rate, whereas 'frail' ( $\mathrm{mFI} \geq 0.27$ ) was significantly correlated with increased 1 -year mortal-

Table 2. Descriptive univariate analysis of traumatic C2 fractures in patients aged 65 or above between 2010 and 2016 at a single institution

\begin{tabular}{|c|c|}
\hline Characteristic & Value \\
\hline Mean age & $80.6 \pm 8.5(65-97)$ \\
\hline \multicolumn{2}{|l|}{ Gender } \\
\hline Female & $42(60)$ \\
\hline Male & $28(40)$ \\
\hline \multicolumn{2}{|l|}{ Ethnicity } \\
\hline European & $62(88)$ \\
\hline Maori/Pacific Islander & $7(10)$ \\
\hline Asian & $1(1.4)$ \\
\hline Mean Charlson Comorbidity Index & $5.3 \pm 2.2(2-10)$ \\
\hline Mean modified Frailty Index & $0.21 \pm 0.15(0-0.55)$ \\
\hline \multicolumn{2}{|l|}{ Mechanism of injury } \\
\hline Fall from standing height & $52(74.3)$ \\
\hline High speed MVC & $12(17.1)$ \\
\hline Low speed MVC & $4(5.7)$ \\
\hline Pedestrian vs. car & $2(2.9)$ \\
\hline Abnormal neurology on presentation & $8(11.4)$ \\
\hline \multicolumn{2}{|l|}{ Treatment modality } \\
\hline Hard collar & $38(54.3)$ \\
\hline Soft collar & $10(14.3)$ \\
\hline Halo-vest & $5(7.1)$ \\
\hline Minerva brace & $8(11.4)$ \\
\hline Traction/tongs & $1(1.4)$ \\
\hline Surgery & $8(11.4)$ \\
\hline Average length of stay & $10.4 \pm 12.5(0-77)$ \\
\hline Readmission within 90 days & $20(28.5)$ \\
\hline \multicolumn{2}{|l|}{ Mortality } \\
\hline 30-Day & $10(14.3)$ \\
\hline 1-Year & $25(35.7)$ \\
\hline
\end{tabular}

Values are presented as mean \pm standard deviation (range) or number (\%).

MVC, motor vehicle collision. ity $(R=0.538, p=001)$. Albumin and hemoglobin levels were inversely correlated with both the 30-day $(R=-0.286$, $p=0.016 ; R=-0.373, p=0.001)$ and 1 -year $(R=-0.350$, $p=0.003 ; R=-0.319, p=0.007)$ mortality rates.

\section{Multivariate analysis}

Forward stepwise logistic regression modeling adjusting for age, gender, CCI, mFI, hemoglobin level, lymphocyte count, albumin, and creatinine determined that increased CCI (odds ratio [OR], 1.614; 95\% confidence interval [CI], 1.145-2.276; $p=0.006$ ), reduced hemoglobin level (OR, 0.919 ; $95 \%$ CI, $0.866-0.975 ; p=0.005)$, and reduced creatinine level (OR, 0.989; 95\% CI, 0.976-1.002; $p=0.095$ ) were the highest predictors of 30-day mortality rates, reaching inclusion into the final model $\left(R^{2}=0.431\right)$. Increased CCI (OR, 2.875; 95\% CI, 1.579-5.235; $p=0.001$ ), increased age (OR, 1.154; 95\% CI, 1.003-1.329; $p=0.045)$, reduced albumin level (OR, 0.762; 95\% CI, 0.621-0.936; $p=0.01$ ), and female gender (OR, 11.188; 95\% CI, 1.307-95.795; $p=0.028$ ) were predictors of 1 -year mortality rate, reaching inclusion to the final model $\left(R^{2}=0.762\right)$ (Table 4$)$. By contrast, mechanism of injury, fracture type, displacement, associated injuries, presence of neurological deficits, and treatment modality were not correlated with mortality rates at either 30 days or 1 year in the analysis.

\section{Discussion}

The present study aimed to determine the 30-day and 1-year mortality rates in elderly patients with traumatic C2 fractures and identify risk factors for mortality. A significant 1 -year mortality rate of $35.7 \%$ was noted, similar to that of previous reports reported in elderly patients with hip fractures [2,19-22].

Although increased comorbidity status and frailty were associated with mortality rates at both 30 days (low positive correlation) and 1 year (moderately positive) in the bivariate analysis, multivariate analysis determined that, of the two measures, only CCI was a significant predictor of mortality rate at both time points. The present study is the first, to the best of our knowledge, to show that CCI independently predicts mortality rate in elderly patients with C2 fractures. Several studies have reported that comorbidity is a reliable predictor of mortality rates and postoperative outcomes in a number of pathologies, including elderly patients with femur fractures, both proxi- 
Table 3. Bivariate analysis of 30-day and 1-year mortality rates, 90-day readmission rates and length of stay

\begin{tabular}{|c|c|c|c|c|c|c|c|c|}
\hline \multirow{2}{*}{ Variable } & \multicolumn{2}{|c|}{ 30-Day mortality } & \multicolumn{2}{|c|}{ 1-Year mortality } & \multicolumn{2}{|c|}{ 90-Day readmission } & \multicolumn{2}{|c|}{ Length of stay } \\
\hline & $R$ & $p$-value & $R$ & $p$-value & $R$ & $p$-value & $R$ & $p$-value \\
\hline Charlson Comorbidity Index & 0.367 & 0.002 & 0.690 & $<0.001$ & 0.142 & 0.241 & -0.204 & 0.090 \\
\hline $\mathrm{mFl}^{\mathrm{a})}$ & 0.313 & 0.008 & 0.583 & $<0.001$ & 0.331 & 0.005 & -0.075 & 0.537 \\
\hline Pre-frail & -0.140 & 0.371 & 0.063 & 0.689 & 0.122 & 0.437 & 0.128 & 0.412 \\
\hline Frail & 0.203 & 0.228 & 0.538 & 0.001 & 0.320 & 0.053 & 0.020 & 0.905 \\
\hline Haemoglobin (g/L) & -0.373 & 0.001 & -0.319 & 0.007 & -0.070 & 0.951 & -0.170 & 0.161 \\
\hline Lymphocyte count & 0.077 & 0.524 & 0.037 & 0.759 & -0.133 & 0.271 & -0.032 & 0.792 \\
\hline Albumin (g/L) & -0.286 & 0.016 & -0.350 & 0.003 & -0.101 & 0.405 & -0.082 & 0.498 \\
\hline Creatinine $(\mu \mathrm{mol} / \mathrm{L})$ & 0.061 & 0.616 & 0.233 & 0.053 & 0.053 & 0.661 & 0.037 & 0.759 \\
\hline
\end{tabular}

Significant results in bold type.

$\mathrm{mFI}$, modified Frailty Index.

alPre-frail: $\mathrm{mFI}, 0.09-0.18$; frail: $\mathrm{mFI} \geq 0.27$.

Table 4. Multivariate analysis of 30-day and 1-year mortality using forward stepwise regression modelling

\begin{tabular}{|c|c|c|c|c|}
\hline \multirow{2}{*}{ Variables entered into analysis } & \multicolumn{2}{|c|}{ 30-Day mortality (final model) } & \multicolumn{2}{|c|}{ 1-Year mortality (final model) } \\
\hline & OR $(95 \% \mathrm{CI})$ & $p$-value & OR $(95 \% \mathrm{CI})$ & $p$-value \\
\hline Age & - & 0.181 & 1.154 (1.003-1.329) & 0.045 \\
\hline Female gender & - & 0.453 & 11.188 (1.307-95.795) & 0.028 \\
\hline Charlson Comorbidity Index & $1.614(1.145-2.276)$ & 0.006 & $2.875(1.579-5.235)$ & 0.001 \\
\hline Modified Frailty Index & - & 0.797 & - & 0.59 \\
\hline Haemoglobin & 0.919 (0.866-0.975) & 0.005 & - & 0.061 \\
\hline Lymphocyte count & - & 0.658 & - & 0.098 \\
\hline Albumin & - & 0.318 & $0.762(0.621-0.936)$ & 0.01 \\
\hline Creatinine & $0.989(0.976-1.002)$ & 0.095 & - & 0.851 \\
\hline$R^{2 \mathrm{a})}$ & 0.431 & & 0.762 & \\
\hline
\end{tabular}

$\mathrm{OR}$, odds ratio; $\mathrm{Cl}$, confidence interval.

${ }^{\text {a) }}$ Coefficient of determination.

mal and distal, spinal disorders, and undergoing spinal surgery [5,6,23-25]. Therefore, the present study reaffirms that pre-injury disease burden and the comorbidity status adversely affect survival rate in the setting of elderly C2 fractures. Of readmissions, almost none were associated with fracture or injury itself, but rather medical conditions including urinary tract infections, lower respiratory infections, or gastrointestinal complaints (e.g., constipation).

The present study used the mFI to examine frailty as a risk factor, focusing on the pre-injury functional dependance and physical vulnerability of the patient to medical insults or injuries. It was hypothesized that $\mathrm{mFI}$ is a significant predictor as, similar to the CCI, it has been linked to morbidity and mortality rates in similar settings [913], and was shown to correlate with mortality rate in the initial bivariate analysis performed. However, the results of the multivariate regression model failed to show $\mathrm{mFI}$ as a risk factor.

Bajada et al. [3] reported a low hemoglobin level as a predictor of mortality rate, and the results of the present study confirm this association. Anemia likely serves a role as an indicator of a chronic disease state and consequently a reduced physiological reserve. In addition, the present study found that low serum albumin was another significant risk factor associated with 1-year mortality rate. Previously, Chung et al. [26] showed that increasing severity of malnutrition, as defined by the level of albu- 
min deficiency, predicts progressively poorer 30-day outcomes in patients undergoing hip fracture surgery. Either reduced hepatic protein synthesis or a malnourished state can result in lowered serum albumin and should prompt appropriate multidisciplinary input.

Treatment of C2 fractures at our center over the study period was predominantly conservative, with only $11.4 \%$ of patients undergoing surgical management, similar to studies from centers in other geographic regions [22,27]. Although Pearson et al. [22] reported reduced 30-day and 1-year mortality rates with surgical stabilization in their Medicare cohort, the mortality rates in the cohort examined in the present study do not appear to correlate with treatment modality. This may represent the range of physiologic states encountered in patients aged $>65$ years and their consequent fitness for an invasive procedure, a key consideration in determining optimal fracture treatment.

There were limitations to the present study. It was retrospective in nature, and any retrospective analysis is influenced by the accuracy of prior documentation. The present study included all C2 fracture patterns and all mechanisms of injury and treatment modalities. With the retrospective study reviewing cases, which occurred over a period of 7 years, and with multiple surgeons being involved, there is a potential for variation in treatment, even for similar fracture patterns. However, it is difficult to control for surgical indication and choice of treatment due to the above findings, and there may be additional confounding factors; however, inherent individual differences exist even in similar injury patterns (e.g., level of comorbidities and fitness for surgery), which cannot be easily controlled. Although this creates a level of heterogeneity in the cohort, it also means the results are more broadly applicable. Indeed the fracture patterns and mechanism in this cohort did not correlate with any of the outcome measures in the analyzes. The trend of treatment at our center was toward nonsurgical treatment for most cases of C2 fractures, and hard or rigid orthoses were avoided in those at risk of pressure sores and aspiration pneumonia.

\section{Conclusions}

The results of the present study reaffirm the significant mortality associated with $\mathrm{C} 2$ fractures in the geriatric population, with a rate approaching $40 \%$ at 1 year. It also established that comorbidity status, as indicated by the CCI, low serum hemoglobin, low serum albumin, increased age, and female gender were associated with 1 -year mortality rate.

\section{Conflict of Interest}

No potential conflict of interest relevant to this article was reported.

\section{References}

1. Ryan MD, Henderson JJ. The epidemiology of fractures and fracture-dislocations of the cervical spine. Injury 1992;23:38-40.

2. Venkatesan M, Northover JR, Wild JB, et al. Survival analysis of elderly patients with a fracture of the odontoid peg. Bone Joint J 2014;96-B:88-93.

3. Bajada S, Ved A, Dudhniwala AG, Ahuja S. Predictors of mortality following conservatively managed fractures of the odontoid in elderly patients. Bone Joint J 2017;99-B:116-21.

4. Tashjian RZ, Majercik S, Biffl WL, Palumbo MA, Cioffi WG. Halo-vest immobilization increases early morbidity and mortality in elderly odontoid fractures. J Trauma 2006;60:199-203.

5. Kirkland LL, Kashiwagi DT, Burton MC, Cha S, Varkey P. The Charlson Comorbidity Index score as a predictor of 30-day mortality after hip fracture surgery. Am J Med Qual 2011;26:461-7.

6. Neuhaus V, King J, Hageman MG, Ring DC. Charlson comorbidity indices and in-hospital deaths in patients with hip fractures. Clin Orthop Relat Res 2013;471:1712-9.

7. Fried LP, Tangen CM, Walston J, et al. Frailty in older adults: evidence for a phenotype. J Gerontol A Biol Sci Med Sci 2001;56:M146-56.

8. Boeckxstaens P, Vaes B, Legrand D, Dalleur O, de Sutter A, Degryse JM. The relationship of multimorbidity with disability and frailty in the oldest patients: a cross-sectional analysis of three measures of multimorbidity in the BELFRAIL cohort. Eur J Gen Pract 2015;21:39-44.

9. Youngerman BE, Neugut AI, Yang J, Hershman DL, Wright JD, Bruce JN. The modified frailty index and 30-day adverse events in oncologic neurosurgery. J Neurooncol 2018;136:197-206.

10. Tomlinson SB, Piper K, Kimmell KT, Vates GE. Pre- 
operative frailty score for 30-day morbidity and mortality after cranial neurosurgery. World Neurosurg 2017;107:959-65.

11. Schaller MS, Ramirez JL, Gasper WJ, Zahner GJ, Hills NK, Grenon SM. Frailty is associated with an increased risk of major adverse cardiac events in patients with stable claudication. Ann Vasc Surg 2018;50:38-45.

12. Ali R, Schwalb JM, Nerenz DR, Antoine HJ, Rubinfeld I. Use of the modified frailty index to predict 30day morbidity and mortality from spine surgery. J Neurosurg Spine 2016;25:537-41.

13. Miller EK, Neuman BJ, Jain A, et al. An assessment of frailty as a tool for risk stratification in adult spinal deformity surgery. Neurosurg Focus 2017;43:E3.

14. Charlson ME, Pompei P, Ales KL, MacKenzie CR. A new method of classifying prognostic comorbidity in longitudinal studies: development and validation. J Chronic Dis 1987;40:373-83.

15. Karam J, Tsiouris A, Shepard A, Velanovich V, Rubinfeld I. Simplified frailty index to predict adverse outcomes and mortality in vascular surgery patients. Ann Vasc Surg 2013;27:904-8.

16. Thongprayoon C, Cheungpasitporn W, Kashani K. Serum creatinine level, a surrogate of muscle mass, predicts mortality in critically ill patients. J Thorac Dis 2016;8:E305-11.

17. Jellinge ME, Henriksen DP, Hallas P, Brabrand M. Hypoalbuminemia is a strong predictor of 30-day allcause mortality in acutely admitted medical patients: a prospective, observational, cohort study. PLoS One 2014;9:e105983.

18. Bajada S, Smith A, Morgan D. Pre-operative nutritional serum parameters as predictors of failure after internal fixation in undisplaced intracapsular proxi- mal femur fractures. Injury 2015;46:1571-6.

19. Schoenfeld AJ, Bono CM, Reichmann WM, et al. Type II odontoid fractures of the cervical spine: do treatment type and medical comorbidities affect mortality in elderly patients? Spine (Phila Pa 1976) 2011;36:879-85.

20. Cooper Z, Mitchell SL, Lipsitz S, et al. Mortality and readmission after cervical fracture from a fall in older adults: comparison with hip fracture using national Medicare data. J Am Geriatr Soc 2015;63:2036-42.

21. Harris MB, Reichmann WM, Bono CM, et al. Mortality in elderly patients after cervical spine fractures. J Bone Joint Surg Am 2010;92:567-74.

22. Pearson AM, Martin BI, Lindsey M, Mirza SK. C2 vertebral fractures in the Medicare population: incidence, outcomes, and costs. J Bone Joint Surg Am 2016;98:449-56.

23. Arrigo RT, Kalanithi P, Cheng I, et al. Predictors of survival after surgical treatment of spinal metastasis. Neurosurgery 2011;68:674-81.

24. Dimar JR, Fisher C, Vaccaro AR, et al. Predictors of complications after spinal stabilization of thoracolumbar spine injuries. J Trauma 2010;69:1497-500.

25. Streubel PN, Ricci WM, Wong A, Gardner MJ. Mortality after distal femur fractures in elderly patients. Clin Orthop Relat Res 2011;469:1188-96.

26. Chung AS, Hustedt JW, Walker R, Jones C, Lowe J, Russell GV. Increasing severity of malnutrition is associated with poorer 30-day outcomes in patients undergoing hip fracture surgery. J Orthop Trauma 2018;32:155-60.

27. Chen YR, Boakye M, Arrigo RT, et al. Morbidity and mortality of C2 fractures in the elderly: surgery and conservative treatment. Neurosurgery 2012;70:10559. 